\title{
Comprehensive Performance Test of Dobby and Analysis System
}

\author{
Honghuan Yin ${ }^{1, ~ a, ~ H o n g b i n ~ Y u ̈, ~ b, ~ Y o n g l i a n g ~} \mathrm{Jin}^{2, \mathrm{c}}$, Xuekui $\mathrm{Li}^{2, \mathrm{c}}$ \\ ${ }^{1}$ School of Mechanical Engineering, Tianjin Polytechnic University, Tianjin, 300387, China \\ 2Jiangsu Jinlong Technology Co., LTD, Jiangsu, 215505, China \\ ayhh201184@163.com, bhongbinyu@aliyun.com, cjinlong@long-xing.net
}

Keywords: Dobby, Textile, Comprehensive performance, Detection.

\begin{abstract}
Dobby, as a kind of commonly used fabric system and shuttleless loom, is applied to all kinds of weaving factory. Compared with the tappet loom, it could weave more complex flower fabric. Dobby fabric play a key role in the process of research and development of new varieties and batch manufacturing. The working performance of dobby influenced remarkably the yield and quality of the products. However, at present, the investigation about dobby performance test is rare and there are not a complete test equipment to produce dobby for many manufacturers, which lead to the dobby produced can't reach the criteria and restrict the development of China's textile industry. The comprehensive performance test system of dobby would ensure the performance of dobby meet the requirement.
\end{abstract}

\section{Introduction}

Detection technology of dobby is relatively backward in China, which seriously restrict the development and production of dobby. The dobby produced could not guarantee that every action is accurate. The dobby shedding mechanism harness motion error in the process of weaving which lead to malposition of fabric pattern and affect production.

As an important part of weaving machinery, Dobby has a key influence to efficiency and quality of loom. High speed rotary dobby was the most advanced models and widely used in the textile weaving due to its ingenious structure design and advanced manufacturing technology. Although the application of high speed rotary dobby has been more than 20 years in Chinese market, the core technology and the main market were still occupied by foreign enterprises [1]. The study about rotary dobby therefore could result in promotion of Chinese textile technology and development of the textile industry which has great theoretical significance and application prospect.

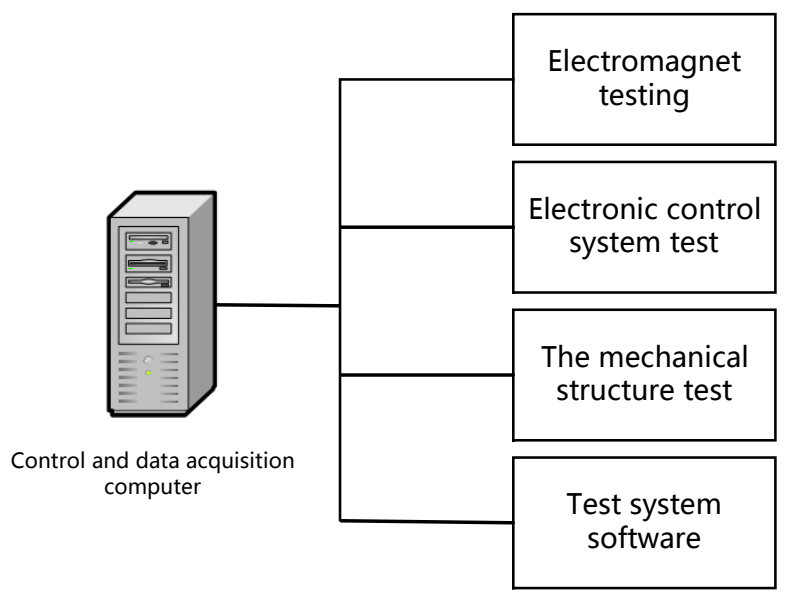

Fig. 1 Comprehensive performance test system block diagram 
The objective of this work was to study the reliability and basic function of electronic dobby. It would provide an effective method to test electronic dobby, help the producers test its performance and improve technology, produce qualified electronic dobby and promote the development of the textile industry. The content of test includes four parts: electromagnet test, electronic control system test, mechanical structure and test system software which were shown in Fig. 1.

\section{Summary of Electromagnet Testing System}

\subsection{The Meaning of Electromagnet Testing}

As the linkage of controller and harness arm, the performance of electromagnet directly affect the motion rules of harness arm and frame, making the weave pattern produced did not meet the requirement of design and influencing the fabric appearance. Air gap width, working voltage and bypass electromagnet would influence the motion tracks of harness arm. The electromagnetic properties was detected under the specific condition by electromagnet testing system. The electromagnet suction release time and magnetic table were monitored at the same condition. It is found that abnormal phenomenon appeared for electromagnet in long-term work, corresponding measures should be taken to repair or replace the electromagnet.

\subsection{The Electromagnet Testing}

Electromagnet detection system mainly detect two department: process analysis and electromagnet strength test [2].

In terms of process analysis, the commonly used electromagnet is clapper type with model $\mathrm{E}$ (Fig.2 and Fig. 3).
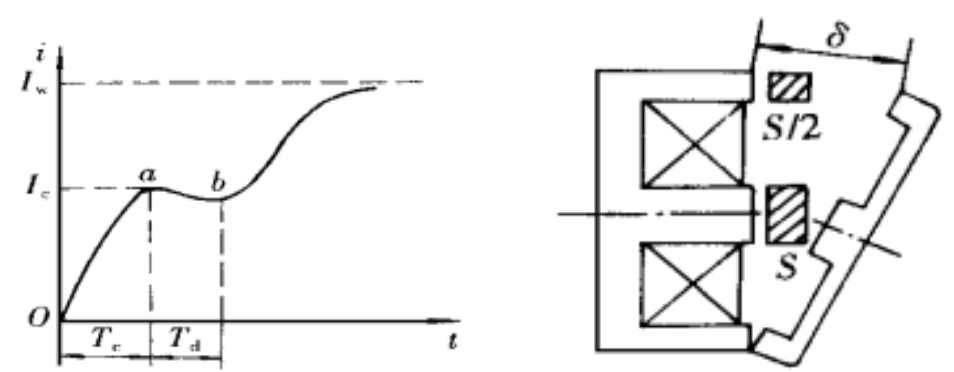

Fig. 2 E jewels electromagnet

Fig. 3 Electromagnet suction characteristic curve

The suction characteristic was shown at Eq. 1.

$$
\mathrm{F}=(\mathrm{IN})^{2} \mu_{0} \frac{\mathrm{s}}{\delta^{2}}
$$

Where I is the coil current; $\mathrm{N}$ is the coil number of turns; $\mu_{0}$ is vacuum magnetic permeability; $\delta$ is the gap.

After supplying the power, the current through the coil and magnetic flux increased gradually before suction overcame the resistance and make the armature move. The circuit equation is shown at Eq. 2.

$$
\mu=\operatorname{ir}+\frac{d \psi}{d t}
$$

Where $\mu$ is terminal voltage; $i$ is electric current; $r$ is the coil resistance; $\psi$ is magnetic chain.

Assuming that the circuit magnet of magnetic is linear, magnetic chain $(\psi)$ was proportional to the current(i) as the armature is not moving. Moreover, the inductance (L) in the circuit is constant, then the formula can be simplified to Eq. 3.

$$
\mu=\operatorname{ir}+\mathrm{L} \frac{\mathrm{di}}{\mathrm{dt}}
$$


Stage oa was the touch stage. Armature started to move after point a (set at current $t$ is $I_{c}$ for his time. and $T_{c}$ is called touch time). The air gap reluctance change when Armature moved. The self inductance $\mathrm{L}$ of coil also changed shown at Eq. 4.

$$
\frac{\mathrm{d} \psi}{\mathrm{dt}}=\frac{\mathrm{d}(\mathrm{Li})}{\mathrm{dt}}=\mathrm{L} \frac{\mathrm{di}}{\mathrm{dt}}+\mathrm{i} \frac{\mathrm{dL}}{\mathrm{dt}}
$$

So the Eq. 2 changed to Eq. 5

$$
\mu=\operatorname{ir}+\mathrm{L} \frac{\mathrm{di}}{\mathrm{dt}}+\mathrm{i} \frac{\mathrm{dL}}{\mathrm{dt}}
$$

Current began to decline until completely closed due to the increasing of $\mathrm{i} \frac{\mathrm{dL}}{\mathrm{dt}}$ which was shown at ab stage in Fig. 2. Where $T_{d}$ is the time for moving, since then, $L$ no longer change, and gradually increase to stable current value $I_{w}$. The current remain constant when $\frac{d i}{d t}=0$, then the steady current $I_{w}$ could be calculated by the following equation: $I_{w}=\frac{\mu}{r}$. The absorption of electromagnet pull in time is: $\mathrm{T}_{\mathrm{x}=} \mathrm{T}_{\mathrm{c}}+\mathrm{T}_{\mathrm{d}}$.

The other test was electromagnet strength, the PCB board equipped with linear hall element was installed on the surface of electromagnet. Every hall element corresponding to each electromagnet. Relay was used to provide working voltage to electromagnet and control each electromagnet which can work independently and steadily.

When every electromagnet worked steadily, hall element will produce the corresponding electrical signals. The total controller acquired these electrical signals and the superficial magnetic field was calculated by the linear equation of hall element [3]. The data was transferred to the PC again on the total control system. Finally, the performance of electromagnet is tested (Fig. 4 and Fig. $5)$.

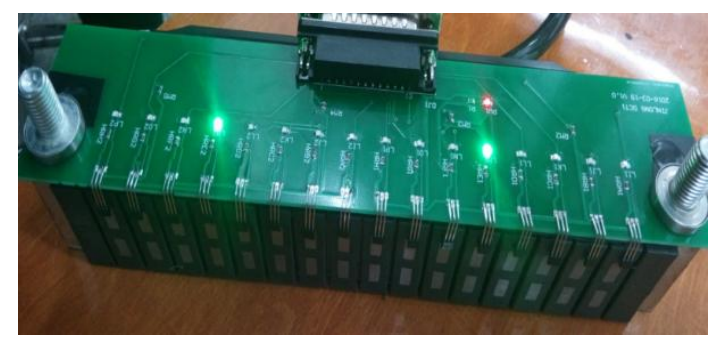

Fig. 4 The principle diagram

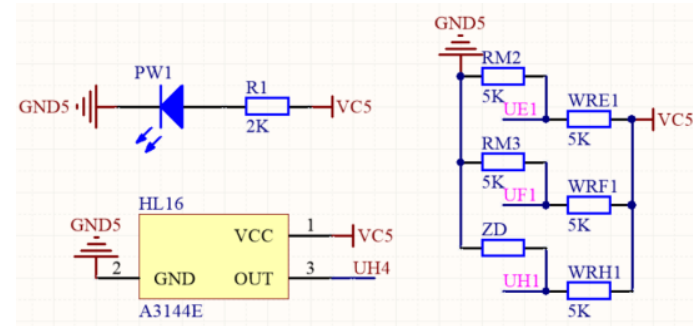

Fig. 5 Electromagnet testing circuit diagram

\section{Reliability Test of Electronic Control System}

As the total control unit of electronic dobby, Electric control system play a key role in the working state of electronic dobby. The nonstable electronic control system would lead to the deviation of input and output signal, influence remarkably the designs and patterns of fabric [4], even damage the dobby. Electric control system test is mainly to test the consistency of the input signal and output signal by comparing the input signal frequency, output signal frequency and time of signal transformation (Fig. 6). 


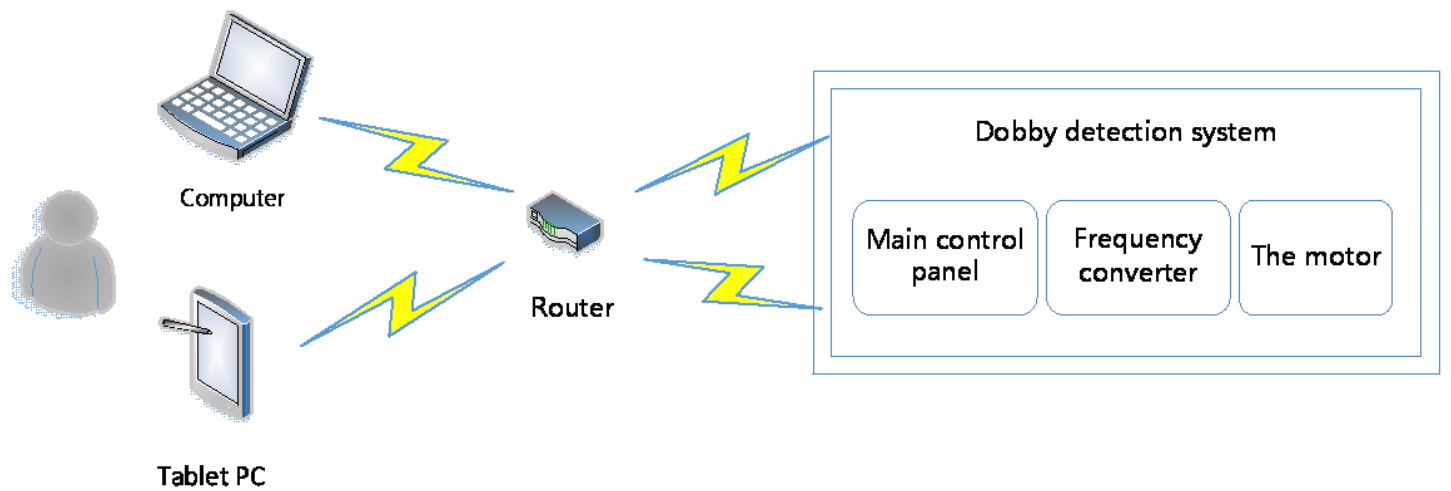

Fig. 6 Electronic control system block diagram

The test method: A complex and known signal was designed by the control panel and used as the input signal electric control system. On the output side, the control panel was applied to collect the output signal. The function curve of output signals was obtained by analyzing the output signal. The variation curve of input and output signal was obtained which provide the information of improvement and validation of dobby electronic control system which help the designers to analyze and solve problems and provide the basis for the improvement of dobby. The rotary dobby of internal institutions can be divided into four modules: electric control equipment module and harness module, solenoid control module, choose all institutions according to different effects of component parts on dobby in the process. Electric control equipment module is composed of single chip microcomputer and a series of electronic components [5] which used to implement the data exchange between computer and software code to control the movement of heald frame set order, to control the design and color of the fabric (Fig. 7).

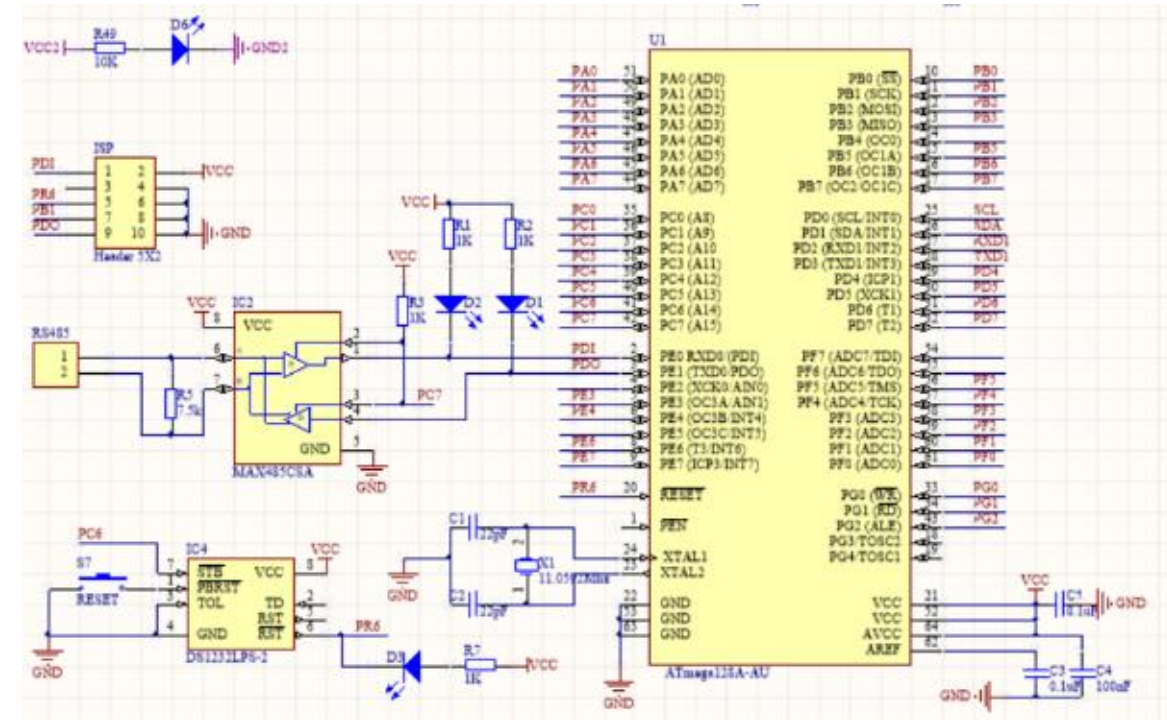

Fig. 7 Electronic control system master control principle diagram

\section{The Mechanical Structure Test}

The mechanical structure of simulation dobby drive heald frame was designed and manufactured. After eliminating the effect of electric control system and the reliability of the electromagnet, the reliability of the mechanical structure of dobby was tested and verificated. The abnormal mechanical structure is found and recorded in the long-term work. The selective control of heald frame motion control. Rotary dobby could only work normally under the combined of lifting mechanism and selecting mechanism. However, the selective control of integrated frame motion was the mechanical 
movement process with high precision. Every procedure must be processed correctly. If one procedure appeared deviation, the timing of the integrated frame motion would be error, then lead to knock-on effect, make all motions of the group heald frame chaos and finally caused the useless of fabric as it could not meet the requirement of design. In order to effectively and selective control the integrated frame motion by rotary dobby, selecting mechanism must be designed to avoid the mechanical corrosion with rotating speed changing mechanism and ensure the accurate cooperation between selecting mechanism and comprehensive extraction (Fig. 8).

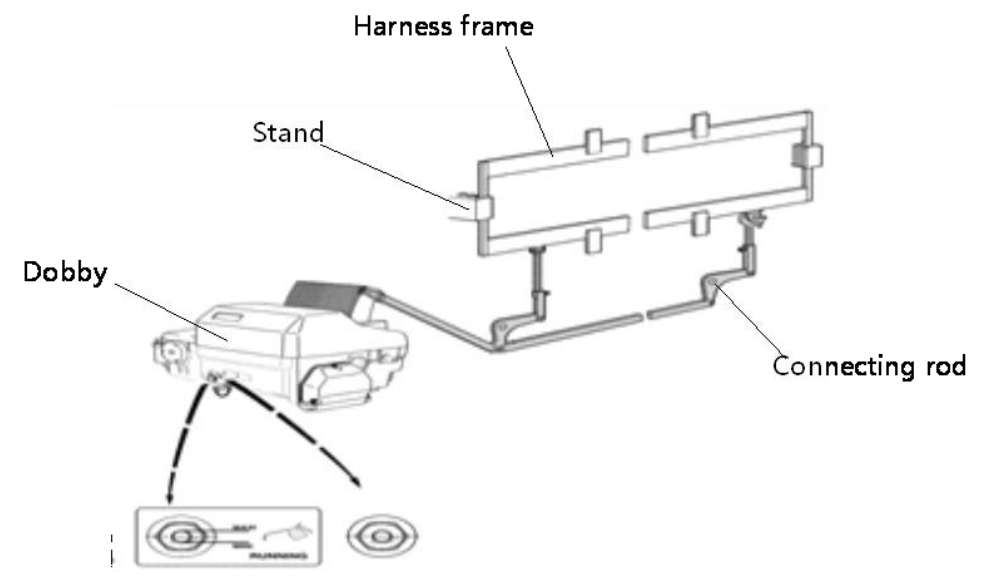

Fig. 8 Test system mechanical structure drawing

Dobby can be processed steadily and orderly during the normal operation, input torque $M$ and output force $\mathrm{F}$ of dobby was recorded by the test system, then process efficiency of dobby was calculated. The transmission performance was evaluated by comparing with standard design. Input torque $\mathrm{M}$ is shaft torque of electronic dobby and measured by shaft torque sensor installed in input shaft dobby [7]; $\mathrm{F}$ is output force of dobby harness arm and obtained by harness tension sensors installed in the arm on the connecting rod. Input torque and output power measurement combined with the speed of the swing arm, work efficiency of dobby can be calculated by the formula of mechanical efficiency. The input power can be obtained by torque sensor measurement of torque and rotational speed $\mathrm{N}$ which shown at the following equation: $\mathrm{M}=9550 \mathrm{P} / \mathrm{N}$.

\section{Software of Test System}

Comprehensive performance test and analysis system of dobby was developed. This system was based on virtual instrument technology and take the advantage of the powerful processing function of computer and highly integrated data acquisition card. The test system was established by virtual instrument software programming to be suitable for textile machinery. This test system can measure fastly and shown realtime the input and output rotational speed, the output torque of dobby, input and output power, mechanical efficiency, vibration, noise, temperature parameters, such as the fast measurement and real-time display. Furthermore, the data could be saved for offline processing, analysis and calculation and so on. This test system could complete the required test functions, also implements the testing system of digitalization, graphical and testing automation and networking, has friendly interface, easy operation, high cost performance and so on at the same time. Testing sensor in the test system is the role of measurement data, and in the process of dobby running real-time feedback test information. The effect of monitoring dobby prototype operation condition is stable. Installation of test sensor by other devices connected to the test software, the monitoring process is real-time, stable and efficient. Test of sensor used in this test system mainly includes the torque sensor, pressure sensor and temperature sensor. According to the test system design requirements, a complete 
set of dobby test system includes three parts: sensors, data acquisition device, virtual software (Fig. 9).

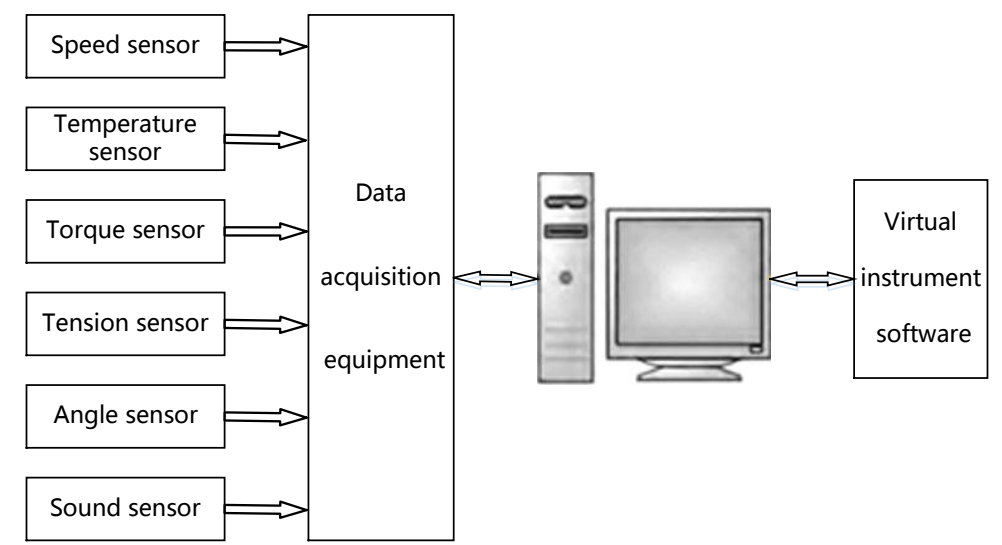

Fig. 9 Diagram of the test system software

\section{Conclusion}

In the increasingly grim situation, the excellent institutions characteristics of dobby has become the focus of market and enterprise most. Dobby manufacturing technology is relatively backward in China, the domestic enterprises exist problems such as small scale, insufficient technical innovation ability which severely restricted the rotating at high speed and the development of electronic dobby research field. How to ensure that every action accurate, in order to avoid because of dobby shedding mechanism in the process of normal weaving a harness motion error, lead to defective fabric (wrong flowers - fabric pattern misplacement), so for rotary dobby manufacturers in machine must undergo strict performance tests before they leave.

Dobby comprehensive performance test and analysis system, the function is to complete the dobby work comprehensive performance test, reliability test and the basic function test. Through the test can verify dobby design and assembly quality, through theoretical analysis and experimental verification for dobby optimization design. In addition, dobby comprehensive performance test and analysis system can provide testing for dobby manufacturers and users.

\section{References}

[1] Yanhong Yuan. The principle of rotary with electronic dobby loom to cooperate. Silk. Vol. 08 (2000), p. 24-25.

[2] Qiuming Shi. Research on the dynamic characteristic of direct current electromagnet. Automation Instrument. Vol. 28(2007) No. 05, p. 20-23.

[3] Yueyang Guo, Ruiqi Chen. Electronic dobby electromagnet suction process and speed up. Shanghai Textile Science and Technology. Vol. 29(2001) No. 05, p. 27-29.

[4] Chunlei Liu: High speed rotary dobby mechanism analysis and design (Master's Thesis, Zhejiang Sci-Tech University, china 2012). p. 1.

[5] Qixia Wang:Rotating electronic dobby selected ensemble system analysis and design(Master's Thesis, Zhejiang Sci-Tech University, china 2013). p. 1.

[6] Bing $\mathrm{Hu}$, Chun Zhang. Kinematics analysis of the type 2861 electronic dobby shedding mechanism. Mechanical. Vol. 34(2007) No. 03, p. 32-34.

[7] Yueyang Guo. A new type of microprocessor controlled positive dobby. Indian Journal of Fibre \& Textile Research. Vol. 28 (2003), p. 275-280. 